\title{
Factores que influyen en la deserción de los alumnos del primer ciclo de educación a distancia en la Escuela de Administración de la Universidad Señor de Sipán. Períodos académicos 2011-1 al 2013-1: lineamientos para disminuir la deserción
}

\author{
MIGUEL ANGEL RUIZ PALACIOS \\ Universidad César Vallejo - Perú \\ Universidad Privada del Norte - Perú
}

Recibido el 01-08-2016; primera evaluación el 31-07-2017; segunda evaluación el 01-10-2017; aceptado el 15-10-2017

\section{Resumen}

En el presente trabajo se investiga los factores que influyen en la deserción de los estudiantes universitarios del primer ciclo de educación a distancia, pertenecientes a la Escuela de Administración de la Universidad Señor de Sipán, entre los semestres 2011-I y 2013-I. Del mismo modo se aborda los factores individuales, socioeconómicos, académicos y finalmente los institucionales.

Dentro de los factores individuales, el que más influyó en la deserción fue la falta de tiempo dedicado al estudio ( $53 \%$ del total de encuestados). Respecto a los factores socioeconómicos, como situación laboral, sueldos percibidos, personas a cargo, etc., ninguno de ellos influyó en la deserción de la mayoría de estudiantes (79,3\% del total de encuestados). Los factores institucionales como la atención recibida, el apoyo administrativo, el entorno virtual, entre otros, no influyeron en la deserción. Finalmente, los factores académicos, como la conformidad con el plan de estudios, la capacitación recibida, etc., no fueron influyentes para la deserción.

Palabras clave: deserción, educación a distancia, factores individuales, factores socioeconómicos, factores institucionales y factores académicos.

\footnotetext{
Magíster en Administración con mención en Gerencia Empresarial por la Universidad Nacional Pedro Ruiz Gallo. Licenciado en Turismo y Negocios. Profesional con experiencia y formación en docencia universitaria. Contacto: migueldecix@gmail.com
} 
Factors affecting the desertion of students of the first cycle of distance education in the school of administration, "University Señor de Sipán». Periods 2011-i to 2013-i: guidelines for reduce the desertion

\section{Abstract}

This paper investigates the factors that influence in the drop-out of university students, of the first cycle of distance education belonging to the administration school of the university Lord of Sipán, between 2011-I to 2013-I. It addresses the individual, socio-economic, academic and finally institutional factors.

Among the individual factors, the one that most influenced the drop-out was the lack of time dedicated to the study (53\% of the total respondents). With regard to socio-economic factors such as: employment status, salaries received, dependents, etc. none of them influenced student dropouts $(79.3 \%$ of all respondents). Among institutional factors such as: care received, administrative support, virtual environment, etc. did not influence desertion. Finally, academic factors such as: conformity with curriculum, training received, etc., were not relevant in desertion.

Keywords: Desertion, distance education, individual factors, socioeconomic factors, institutional factors and academic factors.

Fatores de influência na deserçáo dos alunos do primeiro ciclo de educaçáo a distância na Escola de Administraçáo da Universidad Señor de Sipán. Períodos acadêmicos de 2011-1 a 2013-1: diretrizes para diminuir a deserçáo

\section{Resumo}

Este trabalho investiga os fatores que influenciam o abandono de estudantes universitários do primeiro ciclo de ensino à distância, pertencentes à escola de administração da universidade Senhor do Sipán, entre 2011-I a 2013-I. Aborda os fatores individuais, socioeconômicos, acadêmicos e, finalmente, institucionais.

Entre os fatores individuais, o que mais influenciou o abandono foi a falta de tempo dedicado ao estudo ( $53 \%$ do total de inquiridos). No que diz respeito a fatores socioeconômicos, tais como: status de emprego, salários recebidos, dependentes, etc. nenhum deles influenciou o abandono escolar $(79,3 \%$ de todos os entrevistados). Fatores institucionais, tais como: cuidados recebidos, suporte administrativo, ambiente virtual, etc. não influenciou a deserção. Finalmente, fatores acadêmicos, tais como: conformidade com currículo, treinamento recebido, etc. não eram relevantes em deserção.

Palavras-chave: deserção, educação a distância, fatores individuais, fatores socioeconômicos, fatores institucionais e fatores acadêmicos. 


\section{INTRODUCCIÓN}

La educación a distancia es una modalidad que surge como respuesta a la necesidad de formación y actualización profesional haciendo uso de herramientas tecnológicas como instrumentos para la transmisión de información variada y extensa por parte de la institución educadora hacia el estudiante. La entrega de material impreso, la formación autodidacta, el uso del correo electrónico, las actividades asincrónicas y el almacenamiento de información son algunas características de la modalidad a distancia (Alfonso, 2003). Por otro lado, la educación virtual promueve la comunicación sincrónica en entornos digitales con participación colaborativa, ya que interactúa con los estudiantes, promueve mayor uso de las TIC, transmite y recibe el conocimiento junto al estudiante, siendo, ahora, mediadora y guía del proceso de aprendizaje.

Estas ventajas han hecho que aquellos profesionales de nivel técnico, principalmente, y superior inconcluso se animen por estudiar una carrera universitaria, pues esto implica un desarrollo profesional que traería consigo mejoras laborales, económicas, etc. Sin embargo, pese a estos beneficios se tiene un elevado índice de deserción estudiantil en los programas de educación a distancia, más, aun, en el primer ciclo de estudios. Por ello, en la presente investigación se abarcará los modelos explicativos de deserción estudiantil de Castaño, Gallón, Gómez y Vásquez (2007).

En la región Lambayeque, se cuenta con 11 universidades, aproximadamente, de las cuales tres de ellas (Universidad Señor de Sipán, Los Ángeles de Chimbote y Alas Peruanas), cuentan con educación a distancia, siendo la Universidad Señor de Sipán la de mayor antigüedad en esta modalidad de estudios. Dicho centro de estudios está ubicado en el distrito de Chiclayo y cuenta con una población superior a los 4000 alumnos, aunque cabe mencionar que, por las características del modelo educativo, dichos alumnos son, en su mayoría, de la ciudad de Chiclayo y Lima.

El estudiar a distancia es una facilidad que brindan las universidades para aquellos que, por sus labores, no disponen del tiempo necesario para asistir presencialmente a una universidad y se convierte en una oportunidad de superación profesional en la comodidad del hogar; sin embargo es importante conocer cuáles son las razones por las que muchos alumnos desertan. Recordemos, también, que esta modalidad de estudios demanda de cierto perfil y requisitos para que se pueda culminar satisfactoriamente.

En el Perú se cuenta con el estudio realizado por la Universidad Los Ángeles de Chimbote-Uladech, en la que se identificó las características sociodemográficas de los alumnos que desertaron. Estas muestran que el 63\% fueron hombres 
y el $36,8 \%$ mujeres; el $89,5 \%$ pertenecen a la zona urbana y el 10,5 a la zona rural. Asimismo, el flujo de desertores se encuentra en edad laboral, por lo que concluyen que el motivo de estudiar a distancia es por mejorar sus ingresos y estatus, para el desarrollo profesional y prestigio social. Los resultados obtenidos fueron que la carrera con mayor deserción fue administración $(35,1 \%)$, derecho $(31,6 \%)$ y posteriormente contabilidad y educación.

Es necesario contar con mayor información de otras instituciones que analicen este problema común y muestren propuestas que disminuyan los niveles de deserción, lo cual contribuiría a que el alumno logre sus objetivos profesionales y evitaría la frustración de no alcanzar sus metas de desarrollo, pues existe un perjuicio psicológico del cual la universidad no está exenta (Rizzuto, 2009).

En ese sentido, se realizó la búsqueda de información respecto a la deserción universitaria en países como Colombia, México, Cuba, Argentina, entre otros. Esto ha servido para plantear el problema, los objetivos y la hipótesis de la investigación. Se aplicó un cuestionario a una muestra de 116 estudiantes. Los resultados se procesaron utilizando el programa SPSS versión 20.0 Se analizó los resultados obtenidos, estableciendo las correlaciones para contrastar hipótesis y, finalmente, elaborar las conclusiones y definir las recomendaciones y lineamientos necesarios para disminuir la deserción.

El objetivo del estudio es conocer los factores que influyeron en la deserción de los alumnos del primer ciclo de educación a distancia en la Escuela de Administración de la Universidad Señor de Sipán, entre los períodos académicos 2011-I al 2013-I, para formular lineamientos que contribuyan a disminuir la deserción. Los objetivos específicos buscan conocer los factores individuales, socioeconómicos, institucionales y académicos y su influencia en la deserción del alumno del programa de educación a distancia.

Inicialmente, se realizó un análisis del objeto de estudio, la ubicación de la investigación, cómo surgió el problema, cómo se manifiestó y cuáles fueron sus características y, finalmente, se definió la metodología que se ha usado en el estudio. Así mismo, se cuenta con bases teóricas con relación al objeto de estudio, modelos que explican la deserción de un alumno a distancia (modelo de Tinto, Bean, otros) y el modelo educativo propio de la Universidad Señor de Sipán. Se analizó los resultados obtenidos que indican algunas características más importantes del alumno a distancia, asimismo, se obtuvo el resultado de aquellos factores que determinaron la deserción en los períodos 2011-I al 2013-I. Finalmente, se proporciona los lineamientos que, a consideración del autor, podrán contribuir a disminuir la deserción. 


\section{MATERIALES Y MÉTODOS}

En la presente investigación, se hizo uso de un diseño descriptivo correlacional. Los resultados de la aplicación de los instrumentos fueron analizados según período académico, como se muestra a continuación:

Tabla 1. Alumnos por ciclo académico de estudios

\begin{tabular}{|c|c|c|c|c|c|c|}
\hline \multicolumn{6}{|c|}{ Períodos académicos } & \multirow{2}{*}{ Total } \\
\hline 2011 - I & 2011-II & 2012-0 & 2012-I & 2013-0 & 2013-I & \\
\hline 40 & 41 & 46 & 79 & 75 & 4 & $\mathrm{~N}=285$ \\
\hline 16 & 18 & 15 & 12 & 51 & 4 & $\mathrm{n}=116$ \\
\hline
\end{tabular}

Los estudiantes que participaron pertenecen a la base de datos extraída del programa de educación a distancia. El período de estudio abarcó desde enero de 2014 a diciembre del mismo ańo. El enfoque de investigación es cuantitativo, pues se sustenta en métodos estadísticos utilizando, como técnica, la encuesta y como instrumento el cuestionario.

Se ha utilizado la prueba de chi 2 para determinar la aceptación o rechazo de las hipótesis planteadas en cuanto a la influencia de los factores individuales frente a los institucionales respecto a la deserción estudiantil. El diseño de la investigación fue no experimental de tipo correlacional.

La muestra ha sido seleccionada en relación con los alumnos que, efectivamente, han podido contribuir con resolver el cuestionario de deserción estudiantil por período académico. Los estudiantes que participaron son de las sedes de Lima y Chiclayo (lugares donde se encuentran la mayor cantidad de desertores) y, solamente, aquellos registrados en todos los cursos del primer ciclo de la Escuela de Administración.

Para el análisis de la información, se ha utilizado la estadística inferencial. La población estuvo conformada por todos los alumnos desertores registrados en los cinco cursos del primer ciclo, durante el periodo del 2011-I al 2013-I, correspondiente a 285 estudiantes. El tamańo de muestra se obtuvo de la fórmula de poblaciones finitas, considerando la probabilidad de éxito igual a la probabilidad de fracaso y cuyo error fue del $5 \%$ y un $95 \%$ de confiabilidad. Se obtuvo, como tamaño de muestra, 116 estudiantes. Para seleccionar la muestra, se utilizó el muestreo aleatorio estratificado.

La variable dependiente fue el conjunto de factores individuales del alumno de educación a distancia y la variable independiente la deserción estudiantil.

Esta investigación cumplió con los principios éticos de consentimiento informado, justicia y respeto a la persona; se valoró la integridad y autonomía, asegurando, a su vez, el rigor científico utilizando instrumentos válidos y confiables. 


\section{Resultados}

Los resultados que se muestran a continuación corresponden al objetivo planteado en la investigación: conocer los factores que influyeron en la deserción de los alumnos del primer ciclo de educación a distancia en la Escuela de Administración de la Universidad Señor de Sipán, durante el periodo del 2011-I al 2013-I, para formular lineamientos que contribuyan a disminuir la deserción. Se presentan los resultados de la correlación de variables según el período académico de estudios.

Tabla 2. Factores individuales que influyeron en la deserción

\begin{tabular}{llccccccc}
\hline \multirow{2}{*}{ Período } & Viaje & $\begin{array}{c}\text { Motivos } \\
\text { familiares }\end{array}$ & $\begin{array}{c}\text { Cambio } \\
\text { prioridades }\end{array}$ & Salud & Tiempo & Ninguno & Total \\
\hline $2013-1$ & Recuento & 0 & 0 & 0 & 0 & 2 & 0 & 2 \\
& $\%$ del total &, $0 \%$ &, $0 \%$ &, $0 \%$ &, $0 \%$ & $1,7 \%$ &, $0 \%$ & $1,7 \%$ \\
\hline $2013-0$ & Recuento & 10 & 2 & 0 & 2 & 32 & 4 & 50 \\
& $\%$ del total & $8,6 \%$ & $1,7 \%$ &, $0 \%$ & $1,7 \%$ & $27,6 \%$ & $3,4 \%$ & $43,1 \%$ \\
\hline $2012-1$ & Recuento & 0 & 4 & 4 & 0 & 24 & 2 & 34 \\
& $\%$ del total &, $0 \%$ & $3,4 \%$ & $3,4 \%$ &, $0 \%$ & $20,7 \%$ & $1,7 \%$ & $29,3 \%$ \\
\hline $2012-0$ & Recuento & 2 & 0 & 0 & 0 & 14 & 0 & 16 \\
& $\%$ del total & $1,7 \%$ &, $0 \%$ &, $0 \%$ &, $0 \%$ & $12,1 \%$ &, $0 \%$ & $13,8 \%$ \\
\hline $2011-2$ & Recuento & 2 & 0 & 0 & 0 & 6 & 0 & 8 \\
& $\%$ del total & $1,7 \%$ &, $0 \%$ &, $0 \%$ &, $0 \%$ & $5,2 \%$ &, $0 \%$ & $6,9 \%$ \\
\hline $2011-1$ & Recuento & 0 & 0 & 0 & 0 & 4 & 2 & 6 \\
& $\%$ del total &, $0 \%$ &, $0 \%$ &, $0 \%$ &, $0 \%$ & $3,4 \%$ & $1,7 \%$ & $5,2 \%$ \\
\hline Total & Recuento & 14 & 6 & 4 & 2 & 82 & 8 & 116 \\
& $\%$ del total & $12,1 \%$ & $5,2 \%$ & $3,4 \%$ & $1,7 \%$ & $70,7 \%$ & $6,9 \%$ & $100,0 \%$ \\
\hline
\end{tabular}

Pruebas de chi-cuadrado

\begin{tabular}{llcc}
\hline & Valor & Gl & $\begin{array}{c}\text { Sig. asintótica } \\
\text { (bilateral) }\end{array}$ \\
\hline Chi-cuadrado de Pearson & $34,964^{\mathrm{a}}$ & 25 &, 089 \\
\hline
\end{tabular}

Cuestionario sobre los factores individuales que influyeron en la deserción de los alumnos del primer ciclo de educación a distancia de la Escuela de Administración de la Universidad Señor de Sipán durante los períodos académicos 2011-I al 2013-I. 
Con la prueba de chi-cuadrado, encontramos que la significancia es de 0,089 siendo mayor al nivel de confianza del 95\% (significancia= 0,05 ). Esto nos indica que existe influencia entre los factores individuales y el estudiante que desertó al sistema de educación a distancia durante todo su período de estudios. El factor más influyente es el tiempo con 70,7\%.

Tabla 3. Influencia del apoyo institucional, docente, coordinador y administrativo en la deserción

\begin{tabular}{llccccc}
\hline Período & & $\begin{array}{c}\text { Apoyo } \\
\text { institucional }\end{array}$ & $\begin{array}{c}\text { Apoyo docente o } \\
\text { coordinador }\end{array}$ & $\begin{array}{c}\text { Apoyo } \\
\text { administrativo }\end{array}$ & Ninguno & Total \\
\hline $2013-1$ & Recuento & 0 & 2 & 0 & 0 & 2 \\
& $\%$ del total &, $0 \%$ & $1,7 \%$ &, $0 \%$ &, $0 \%$ & $1,7 \%$ \\
\hline $2013-0$ & Recuento & 2 & 6 & 6 & 36 & 50 \\
& $\%$ del total & $1,7 \%$ & $5,2 \%$ & $5,2 \%$ & $31,0 \%$ & $43,1 \%$ \\
\hline $2012-1$ & Recuento & 6 & 12 & 0 & 16 & 34 \\
& $\%$ del total & $5,2 \%$ & $10,3 \%$ &, $0 \%$ & $13,8 \%$ & $29,3 \%$ \\
\hline $2012-0$ & Recuento & 2 & 0 & 0 & 14 & 16 \\
& $\%$ del total & $1,7 \%$ &, $0 \%$ &, $0 \%$ & $12,1 \%$ & $13,8 \%$ \\
\hline $2011-2$ & Recuento & 2 & 2 & 0 & 4 & 8 \\
& $\%$ del total & $1,7 \%$ & $1,7 \%$ &, $0 \%$ & $3,4 \%$ & $6,9 \%$ \\
\hline $2011-1$ & Recuento & 0 & 0 & 2 & 4 & 6 \\
& $\%$ del total &, $0 \%$ &, $0 \%$ & $1,7 \%$ & $3,4 \%$ & $5,2 \%$ \\
\hline Total & Recuento & 12 & 22 & 8 & 74 & 116 \\
& $\%$ del total & $10,3 \%$ & $19,0 \%$ & $6,9 \%$ & $63,8 \%$ & $100,0 \%$ \\
\hline
\end{tabular}

Pruebas de chi-cuadrado

\begin{tabular}{lccc}
\hline & Valor & Gl & $\begin{array}{c}\text { Sig. asintótica } \\
\text { (bilateral) }\end{array}$ \\
\hline Chi-cuadrado de Pearson & $40,627^{\text {a }}$ & 15 &, 000 \\
\hline
\end{tabular}

Cuestionario sobre los factores institucionales que influyeron en la deserción de los alumnos del primer ciclo de educación a distancia de la Escuela de Administración de la Universidad Señor de Sipán durante los períodos académicos 2011-I al 2013-I. 
Con la prueba chi-cuadrado encontramos que la significancia es de 0,0001 siendo menor que la confianza del $95 \%$ (significancia $=0,05$ ). Esto nos indica que no existe influencia entre los factores institucionales del estudiante que desertó al sistema de educación a distancia durante su período de estudios, el cual está representado por el $63,8 \%$ de los estudiantes.

Tabla 4. Factores socioeconómicos que influyeron en la decisión de desertar

\begin{tabular}{llcccc}
\hline Período & & Incremento de gastos & Inestabilidad laboral & Ninguno & Total \\
\hline $2013-1$ & Recuento & 0 & 0 & 2 & 2 \\
& \% del total &, $0 \%$ &, $0 \%$ & $1,7 \%$ & $1,7 \%$ \\
\hline $2013-0$ & Recuento & 6 & 2 & 42 & 50 \\
& \% del total & $5,2 \%$ & $1,7 \%$ & $36,2 \%$ & $43,1 \%$ \\
\hline $2012-1$ & Recuento & 2 & 0 & 32 & 34 \\
& \% del total & $1,7 \%$ &, $0 \%$ & $27,6 \%$ & $29,3 \%$ \\
\hline $2012-0$ & Recuento & 0 & 2 & 14 & 16 \\
& \% del total &, $0 \%$ & $1,7 \%$ & $12,1 \%$ & $13,8 \%$ \\
\hline $2011-2$ & Recuento & 4 & 4 & 0 & 8 \\
& $\%$ del total & $3,4 \%$ & $3,4 \%$ &, $0 \%$ & $6,9 \%$ \\
\hline $2011-1$ & Recuento & 2 & 2 & 2 & 6 \\
& \% del total & $1,7 \%$ & $1,7 \%$ & $1,7 \%$ & $5,2 \%$ \\
\hline Total & Recuento & 14 & 10 & 92 & 116 \\
& \% del total & $12,1 \%$ & $8,6 \%$ & $79,3 \%$ & $100,0 \%$ \\
\hline
\end{tabular}

Pruebas de chi-cuadrado

\begin{tabular}{lccc}
\hline & Valor & Gl & $\begin{array}{c}\text { Sig. asintótica } \\
\text { (bilateral) }\end{array}$ \\
\hline Chi-cuadrado de Pearson & $49,063^{\mathrm{a}}$ & 10 &, 000 \\
\hline
\end{tabular}

Cuestionario sobre los factores socioeconómicos que influyeron en la deserción de los alumnos del primer ciclo de educación a distancia de la Escuela de Administración de la Universidad Señor de Sipán durante los períodos académicos 2011-I al 2013-I.

Con la prueba chi-cuadrado encontramos que la significancia es de 0,0001 siendo menor que la confianza del $95 \%$ (significancia $=0,05$ ). Esto nos indica que no existe influencia entre los factores socioeconómicos del estudiante que desertó al sistema de educación a distancia durante su período de estudios. El cual está representado por el 79,3\% de los estudiantes. 
Tabla 5. Factores académicos. Calificación del alumno respecto al plan de estudios de la escuela de Administración

\begin{tabular}{llcccc}
\hline & & Regular & Buena & Muy buena & Total \\
\hline $2013-1$ & Recuento & 0 & 2 & 0 & 2 \\
& \% del total &, $0 \%$ & $1,7 \%$ &, $0 \%$ & $1,7 \%$ \\
\hline $2013-0$ & Recuento & 4 & 44 & 2 & 50 \\
& \% del total & $3,4 \%$ & $37,9 \%$ & $1,7 \%$ & $43,1 \%$ \\
\hline $2012-1$ & Recuento & 6 & 28 & 0 & 34 \\
& \% del total & $5,2 \%$ & $24,1 \%$ &, $0 \%$ & $29,3 \%$ \\
\hline $2012-0$ & Recuento & 0 & 16 & 0 & 16 \\
& \% del total &, $0 \%$ & $13,8 \%$ &, $0 \%$ & $13,8 \%$ \\
\hline $2011-2$ & Recuento & 2 & 6 & 0 & 8 \\
& $\%$ del total & $1,7 \%$ & $5,2 \%$ &, $0 \%$ & $6,9 \%$ \\
\hline $2011-1$ & Recuento & 0 & 4 & 2 & 6 \\
& $\%$ del total &, $0 \%$ & $3,4 \%$ & $1,7 \%$ & $5,2 \%$ \\
\hline Total & Recuento & 12 & 100 & 4 & 116 \\
& \% del total & $10,3 \%$ & $86,2 \%$ & $3,4 \%$ & $100,0 \%$ \\
\hline
\end{tabular}

Pruebas de chi-cuadrado

\begin{tabular}{lllc}
\hline & Valor & gl & $\begin{array}{c}\text { Sig. asintótica } \\
\text { (bilateral) }\end{array}$ \\
\hline Chi-cuadrado de Pearson & $24,672^{\mathrm{a}}$ & 10 &, 006 \\
\hline
\end{tabular}

Cuestionario sobre la calificación del plan de estudios de los alumnos del primer ciclo de educación a distancia, en la escuela de administración, de la universidad Señor de Sipán durante los períodos académicos 2011-I al 2013-I.

Con la prueba chi-cuadrado encontramos que la significancia es de 0,006 siendo menor que la confianza del $95 \%$ (significancia $=0,05$ ). Esto nos indica que no existe influencia entre los factores académicos de calificación del plan de estudios del estudiante que desertó al sistema de educación a distancia durante su período de estudios. El cual el $86,2 \%$ considera que es una buena calificación. 
Tabla 6. Calificación de la capacitación recibida al iniciar el primer ciclo

\begin{tabular}{lllllll}
\hline Período & & Muy mal & Mala & Regular & Buena & Total \\
\hline $2013-0$ & Recuento & 2 & 2 & 6 & 20 & 30 \\
& $\%$ del total & $2,9 \%$ & $2,9 \%$ & $8,8 \%$ & $29,4 \%$ & $44,1 \%$ \\
\hline $2012-1$ & Recuento & 0 & 2 & 8 & 6 & 16 \\
& $\%$ del total &, $0 \%$ & $2,9 \%$ & $11,8 \%$ & $8,8 \%$ & $23,5 \%$ \\
\hline $2012-0$ & Recuento & 0 & 0 & 2 & 12 & 14 \\
& $\%$ del total &, $0 \%$ &, $0 \%$ & $2,9 \%$ & $17,6 \%$ & $20,6 \%$ \\
\hline $2011-2$ & Recuento & 0 & 0 & 2 & 0 & 2 \\
& $\%$ del total &, $0 \%$ &, $0 \%$ & $2,9 \%$ &, $0 \%$ & $2,9 \%$ \\
\hline $2011-1$ & Recuento & 2 & 0 & 2 & 2 & 6 \\
& $\%$ del total & $2,9 \%$ &, $0 \%$ & $2,9 \%$ & $2,9 \%$ & $8,8 \%$ \\
\hline Total & Recuento & 4 & 4 & 20 & 40 & 68 \\
& $\%$ del total & $5,9 \%$ & $5,9 \%$ & $29,4 \%$ & $58,8 \%$ & $100,0 \%$ \\
\hline
\end{tabular}

Pruebas de chi-cuadrado

\begin{tabular}{lccc}
\hline & Valor & Gl & $\begin{array}{c}\text { Sig. asintótica } \\
\text { (bilateral) }\end{array}$ \\
\hline Chi-cuadrado de Pearson & $24,945^{\mathrm{a}}$ & 12 &, 015 \\
\hline
\end{tabular}

Cuestionario sobre la calificación respecto a la capacitación recibida hacia los alumnos del primer ciclo de educación a distancia de la Escuela deAadministración de la Universidad Señor de Sipán durante los períodos académicos 2011-I al 2013-I.

Con la prueba chi-cuadrado encontramos que la significancia es de 0,015 siendo menor que la confianza del $95 \%$ (significancia $=0,05$ ). Esto nos indica que no existe influencia los factores académicos en cuanto a la calificación de la capacitación recibida del estudiante que desertó del sistema de educación a distancia durante su período de estudios. Donde el 58,8\% lo considera como buena calificación.

\section{DisCUSIÓN}

En México, como mencionan Contreras y Méndez (2015), en el año 2005, la Universidad Nacional Autónoma de México-UNAM inició una oferta educativa en la modalidad a distancia. Las seis asignaturas preparadas con minucioso cuidado y dedicación fueron el inicio para crecer en esta modalidad, pero tam- 
bién presentaron problemas que aún están vigentes y son parte de una realidad muy similar en la región latinoamericana. Se determinó que, el número de postulantes a dicha modalidad fue más que el doble de la cantidad de vacantes ofrecidas (310 vacantes ofrecidas frente a 773 postulantes). Sin embargo, solo 256 ingresantes lograron hacer sus trámites de inscripción, lo que, en porcentajes, como describen Zubieta, Cervantes y Rojas (2009), significa que, de cada 100 postulantes a estudiar una licenciatura a distancia, solo el 33\% culmina todo el proceso de inscripción.

Para determinar el perfil del alumno a distancia, la UNAM cuenta con información, tales como, edad, estado civil, número de hijos, sueldo, carga familiar, lugar de residencia, entre otros. Con esta información, se puede relacionar los factores económicos, debido a ingresos reducidos; causas laborales, como despidos o cambio de trabajo a otras ciudades; problemas familiares, como salud de parientes cercanos y los problemas familiares internos, otros que influyen en la deserción indirectamente, pues el estudiante da más prioridad a la familia que a los estudios; viajes al extranjero, ya que muchos de ellos no continuaron sus estudios por viajes programados al exterior para mejorar su situación económica; de tipo académico, debido a que cada alumno posee una estructura particular de aprendizaje y conocimientos previos que le dan mayor o menor oportunidad en una modalidad no presencial (Moreno y Cárdenas, 2012). Es importante analizar este aspecto, ya que el bajo rendimiento académico en esta modalidad es característico en más del $80 \%$ del alumnado (Naupari, 2014). Algo importante de rescatar es que, del total de alumnos que desertaron, el $30 \%$ presentó problemas de conexión a internet y poco tiempo para rendir exámenes en línea. Frente a estos problemas, la UNAM planteó soluciones a cada tipo de dificultad, lo que generó una disminución de la deserción. A continuación, se presenta un cuadro resumen del análisis descriptivo realizado:

Tabla 7. Cuadro resumen del análisis descriptivo realizado en la UNAM

\begin{tabular}{|c|c|c|c|c|c|c|c|c|c|c|c|c|c|}
\hline dad & $\mathrm{Se}$ & & $\begin{array}{c}\text { Estado } \\
\text { civil }\end{array}$ & $\begin{array}{c}\text { Sede a } \\
\text { la que } \\
\text { pertenecen }\end{array}$ & $\begin{array}{c}\text { Años } \\
\text { sin } \\
\text { estudiar }\end{array}$ & $\begin{array}{c}\text { Retirado } \\
\text { antes de } \\
\text { partici- } \\
\text { par }\end{array}$ & \begin{tabular}{|c|} 
Horas \\
dedicadas \\
al estudio
\end{tabular} & \begin{tabular}{|c|} 
Causal \\
de retiro \\
funda- \\
mental
\end{tabular} & \begin{tabular}{|c} 
Grado \\
de \\
instr
\end{tabular} & & & $\begin{array}{c}\text { Suel } \\
\text { do }\end{array}$ & $\begin{array}{l}\text { Capa- } \\
\text { cita- } \\
\text { ción }\end{array}$ \\
\hline $\begin{array}{c}20- \\
39 \\
\text { años }\end{array}$ & M & $\mathrm{F}$ & $\mathrm{C}$ & LIM CIX & $\begin{array}{cc}5 & \text { Más } \\
& \text { de } \\
& 10\end{array}$ & $35 \%$ & 0 & Tiempo & \begin{tabular}{|l|} 
Téc- \\
nico
\end{tabular} & Hijos & $\begin{array}{l}\text { s Nin- } \\
\text { guno }\end{array}$ & \multirow[t]{2}{*}{2000} & \multirow[t]{2}{*}{ Buena } \\
\hline $31 \%$ & $67 \%$ & $33 \%$ & $62 \% 29 \%$ & $67 \% 21 \%$ & $26 \% 24 \%$ & & $43 \% 41 \%$ & $53 \%$ & \begin{tabular}{|l|}
$76 \%$ \\
\end{tabular} & $24 \%$ & $66 \%$ & & \\
\hline
\end{tabular}


En Cuba, la Red de Educación a Distancia-EcuRed (2017) realizó una investigación sobre deserción, desde el inicio de los estudios en la modalidad a distancia, en la Universidad de La Habana. Las acciones tomadas para corregir este problema lograron reducir, en promedio, el 54\% de los altos niveles de deserción, según el ciclo académico; sin embargo, para el primer año de estudios, la deserción se mantuvo en un $80 \%$. Tras culminar las dos primeras décadas de haberse iniciado la educación a distancia, se hizo una investigación para conocer las características de los estudiantes que desertaron, tasa de abandono y cuáles fueron los factores que intervinieron en ello. De dicha investigación se obtuvo los siguientes resultados más relevantes: la edad del desertor fue entre 20 y 39 años, solteros y casados con hijos; la principal razón del abandono se relaciona con su vínculo laboral, el mayor porcentaje de desertores se ubica en el primer año y no participaron académicamente. Cerca del $82 \%$ de estudiantes no está preparado para estudiar solo, el $70 \%$ de desertores opinó haber tenido un débil acompañamiento del profesor, falta de información sobre el modelo educativo y poco tiempo para dedicar al estudio. Esta investigación refuerza la concepción de que los factores por los cuales el alumno deserta son, en su mayoría, propios de él (factores individuales); así mismo las características del alumno son similares en ambos análisis: edad entre 20 a 39 años $(81 \%)$, solteros en mayoría $(62,1 \%)$ y casados en menor cantidad $(29,3 \%)$. El principal motivo que les llevó a retirarse fue la falta de tiempo $(53,4 \%)$, la cual, se relaciona directamente al vínculo laboral, coincide además con que la principal deserción se da en el primer año y que un buen porcentaje no participaron académicamente $(34,5 \%)$.

Asimismo, la Universidad Técnica Nacional - UTN ha experimentado un proceso de recolección de información de su alumnado, evaluando aspectos importantes, como la experiencia previa en el manejo de TIC, participación inicial del estudiante, conocimiento del perfil académico, motivación principal por la que postuló y, en cuanto al equipo docente, se fomentó capacitaciones para tutor a distancia, herramientas tecnológicas y metodológicas, etc. (Rama y Bonilla, 2017).

En el Perú, la Universidad Los Ángeles de Chimbote-Uladech identificó las características sociodemográficas de los alumnos que desertaron, siendo el $63 \%$ hombres y $36,8 \%$ mujeres; $89,5 \%$ pertenecen a la zona urbana y 10,5 a la zona rural.

Rescatando los datos más relevantes, se determinó que, según la carrera cursada, la deserción se concentra en la carrera de administración (35,1\%), derecho $(31,6 \%)$ y posteriormente contabilidad y educación. 
Estudiantes desertores según zona de residencia

\begin{tabular}{lcc}
\hline Sexo & $\mathbf{N}^{\circ}$ de estudiantes & $\%$ \\
\hline Urbana & 51 & 89,5 \\
Rural & 6 & 10,5 \\
Total & 57 & 100,00 \\
\hline
\end{tabular}

Fuente: Universidad Alas Peruanas.

La Uladech, según Domínguez y Rama (2013) en cuanto a la educación a distancia, es vista como una oportunidad para crecer profesionalmente. Los alumnos que han desertado, en su mayoría, son varones jóvenes o adultos jóvenes, la mayoría contaba con trabajo remunerado y carga familiar, la mayoría abandonó sus estudios durante los dos primeros años, un porcentaje de ellos reinició sus estudios. Al aplicárseles una encuesta, se concluyó que, en general, los alumnos estaban conformes con los docentes, las evaluaciones, los planes y los programas y, finalmente, los servicios de apoyo académico y administrativo. Las principales causas que determinaron la deserción fueron la falta de tiempo por la dedicación al trabajo y las dificultades económicas. Quintero (2016) menciona que el conjunto de causas individuales, académicas o institucionales que generan la deserción, afectan al individuo en su autoestima y ánimo de retomar sus estudios.

\section{Conclusiones}

Los factores individuales como viajes, motivos familiares, cambio de prioridades, salud y tiempo dedicado al estudio influyeron en la deserción de los estudiantes de educación a distancia. El 70,7\% de los estudiantes consideró la falta de tiempo dedicado al estudio como principal factor.

Los factores socioeconómicos como el incremento de gastos e inestabilidad laboral, no influyeron en la deserción del estudiante de educación a distancia. El 79,3\% de encuestados consideró que ninguno de estos factores ha influido en su deserción.

Respecto a los factores institucionales como el apoyo institucional, apoyo del docente o coordinador de carrera y apoyo administrativo y su influencia en la deserción, encontramos que el sistema de educación a distancia brinda una buena atención institucional y un buen proceso de admisión y matricula y que ninguno de estos factores influyó en la deserción, representado por el $63,8 \%$ de los estudiantes. 
En cuanto a los factores académicos que influyeron en la deserción de los alumnos del sistema a distancia, para el 86,2\% de los estudiantes, no hay influencia en su decisión de desertar y califican al plan de estudios como bueno. Asimismo, el 58,8\% calificó las capacitaciones como buenas.

\section{REFERENCIAS BIBLIOGRÁFICAS}

Alfonso, I. (2003). La educación a distancia. SciELO, 11(1), 3-4. En ACIMED, v.11 n.1 Ciudad de La Habana ene.-feb. 2003. Recuperado de http:// scielo.sld.cu/scielo.php?pid=S102494352003000100002\&script $=$ sci_arttext\&tlng=pt

Castaño, E., Gallón, S., Gómez, K. y Vásquez, J. (2007). Análisis de los factores asociados a la deserción y graduación estudiantil en la Universidad de Antioquia. Lecturas de Economía, (65), 9-36.

Contreras, O. y Méndez, G. (2015). El perfil de los estudiantes de educación a distancia en México. En La educación a distancia en México: una nueva realidad universitaria (pp. 47-64). México: UNAM.

Domínguez, J. y Rama, C. (eds.). (2013). La educación a distancia en el Perú. Chimbote, Perú: Uladech Católica.

Moreno, G. y Cárdenas, M. (2012). Educación a distancia: nueva modalidad, nuevos alumnos. Perfiles de alumnos de Psicología en México. Perfiles Educativos, 34, 118-136.

Naupari, F. (2014). Evaluación del rendimiento académico de estudiantes universitarios en la modalidad presencial y virtual. Apuntes de Ciencia \& Sociedad, 4(1), 69-77. https://doi.org/10.18259/acs.2014007

Quintero, I. (2016). Análisis de las causas de deserción universitaria (tesis de grado). Recuperado de http://repository.unad.edu.co/bitstream/ 10596/6253/1/23783211.pdf

Rama, C. y Bonilla, N. (2017). La educación a distancia y virtual en Costa Rica. Alajuela, Costa Rica: Universidad Técnica Nacional.

Red de Educación a Distancia (2017). La educación a distancia en Cuba. Recuperado de https://www.ecured.cu/Educaci\%C3\%B3n_a_distancia_en_Cuba

Rizzuto, F. (2009). La deserción en la educación superior, motivos y medidas preventivas (tesis de grado). Recuperado de http://web.austral.edu.ar/descargas/ escuela-educacion/tesis-Flavia-Rizzuto.pdf

Zubieta, G. J., Cervantes, P. F. \& Rojas, S. C. (2009). La deserción y el rezago en la educación superior a distancia: signos promisorios en una universidad pública mexicana. Observatorio de la Educación Virtual en América Latina y el Caribe. 